Research Paper

\title{
Melatonin and Vitamin D Orchestrate Adipose Derived Stem Cell Fate by Modulating Epigenetic Regulatory Genes
}

\author{
Sara Santaniello ${ }^{1,2^{*}}$, Sara Cruciani ${ }^{1,2^{*}}$, Valentina Basoli1,2, Francesca Balzano1, Emanuela Bellu1 ${ }^{1}$, Giuseppe \\ Garroni1, Giorgio Carlo Ginesu ${ }^{3}$, Maria Laura Cossu3 3 , Federica Facchin ${ }^{4}$, Alessandro Palmerio Delitala 5 , \\ Carlo Ventura ${ }^{2}$ and Margherita Maioli1,2,6,7凶 \\ 1. Department of Biomedical Sciences, University of Sassari, Viale San Pietro 43/B, 07100 Sassari, Italy; \\ 2. Laboratory of Molecular Biology and Stem Cell Engineering, National Institute of Biostructures and Biosystems - Eldor Lab, Innovation Accelerator, CNR \\ Via Piero Gobetti 101, 40129 Bologna, Italy; \\ 3. General Surgery Unit 2 "Clinica Chirurgica” Medical, Surgical and Experimental Sciences Department, University of Sassari, Viale San Pietro 8, 07100, \\ Sassari, Italy; \\ 4. Department of Experimental, Diagnostic and Speciality Medicine (DIMES), University of Bologna, Via Massarenti 9, 40138 Bologna, Italy; \\ 5. Azienda Ospedaliero-Universitaria di Sassari, Viale San Pietro 8, 07100, Sassari, Italy; \\ 6. Center for Developmental Biology and Reprogramming-CEDEBIOR, Department of Biomedical Sciences, University of Sassari, Viale San Pietro 43/B, \\ 07100, Sassari, Italy; \\ 7. Institute of Genetic and Biomedic Research, Consiglio Nazionale delle Ricerche (CNR), Monserrato, Cagliari, Italy. \\ *These authors contributed equally to this work \\ $\square$ Corresponding author: mmaioli@uniss.it; Tel.: +39 079228277
}

(c) Ivyspring International Publisher. This is an open access article distributed under the terms of the Creative Commons Attribution (CC BY-NC) license (https://creativecommons.org/licenses/by-nc/4.0/). See http://ivyspring.com/terms for full terms and conditions.

Received: 2018.06.05; Accepted: 2018.08.29; Published: 2018.10.20

\begin{abstract}
Melatonin, that regulates many physiological processes including circadian rhythms, is a molecule able to promote osteoblasts maturation in vitro and to prevent bone loss in vivo, while regulating also adipocytes metabolism. In this regard, we have previously shown that melatonin in combination with vitamin $D$, is able to counteract the appearance of an adipogenic phenotype in adipose derived stem cells (ADSCs), cultured in an adipogenic favoring condition. In the present study, we aimed at evaluating the specific phenotype elicited by melatonin and vitamin $D$ based medium, considering also the involvement of epigenetic regulating genes. ADSCs were cultured in a specific adipogenic conditioned media, in the presence of melatonin alone or with vitamin D. The expression of specific osteogenic related genes was evaluated at different time points, together with the histone deacetylases epigenetic regulators, $\mathrm{HDACl}$ and Sirtuins (SIRT) 1 and 2.

Our results show that melatonin and vitamin $D$ are able to modulate ADSCs commitment towards osteogenic phenotype through the upregulation of HDACl, SIRT 1 and 2, unfolding an epigenetic regulation in stem cell differentiation and opening novel strategies for future therapeutic balancing of stem cell fate toward adipogenic or osteogenic phenotype.
\end{abstract}

Key words: melatonin; epigenetic; gene expression; nutraceuticals; cell differentiation; stem cell fate

\section{Introduction}

Human mesenchymal stem cells are undifferentiated cells exhibiting some main feature as self-renewal and differentiation capability, they are located in different areas of our body, organized in specific places called niches, which capture and integrate the environmental signals, influencing stem cell behavior [1]. In particular, Adipose derived stem cells (ADSCs) represent a valuable tool to study stem cell commitment toward different phenotypes, even though they retain a favored adipose commitment [2]. It is increasingly becoming evident that, besides being a neurohormone related to the circadian rhythm, 
melatonin can exert a different number of functions, spanning from mitochondrial activity and the immune system, as well as anti-apoptotic, anti-tumor and anti-ischemic properties [3][4].

Moreover, melatonin exerts different effects also on stem cells, by controlling cell viability, differentiation and apoptosis [5]. The molecular pathway underlying these effects could be mediated by the interaction through melatonin receptors, among which MT1, 2, belonging to the G-protein coupled receptor families, or through a receptor independent manner [6]. Recent papers also describe a role for melatonin as epigenetic modulator [7], by controlling histone deacetylase (HDACs) superfamily, among which Sirtuins (HDAC III) are related to aging and metabolic homeostasis [8]. Sirtuins (SIRT) well represent the epigenetic transduction molecules of different external events, as for example metabolic changes [9]. In particular, these families of enzymes exhibit different activities, along with deacetylation, all requiring NAD+ as coenzyme [10]. In particular, SIRT 1, 3 5, are mostly implicated in metabolic controls, while SIRT2 and SIRT6 control oxidative stress and telomere length, being mainly related to aging processes. Accordingly, it is also described that SIRT expression and activity decline with age [11][12]. Great concentration of free fatty acids, released by adipose tissue, coupled with oxidative stress, directly results in endothelial dysfunction, early atherosclerosis, and coronary artery disease risk factor. SIRT4 is an ADP-ribosyltransferase of $59 \mathrm{kDa}$ variably expressed in liver mitochondria and in skeletal muscle and is associated with homeostasis of glucose/lipid metabolism [13]. Recently, some results demonstrate that melatonin alleviates metabolic inflammation by increasing cellular and exosomal aKG level in adipose tissue [14]. Some data reveal a novel function of melatonin on adipocytes as macrophages communication, suggesting a new potential therapy for this molecule to prevent and treat obesity caused systemic inflammatory disease [14]. Melatonin reduces body weight and inflammation. The mechanism of action of this molecule involve multiple levels, from subcellular to intercellular. Mitochondria may be turned into key inflammation promoters in vascular and adipose tissue, and may become a potential pharmacological target [15]. Melatonin protects against mitochondrial dysfunctions. It also reduces blood pressure and adipose tissue dysfunctions by multiple anti-inflammatory/antioxidant actions and provides potent protection against mitochondria-mediated injury in hypertension and obesity [16].

In a previous study we highlighted that melatonin, together with Vitamin D, was able to counteract adipogenic differentiation, even in an adipogenic milieu created by a specific conditioned medium [17]. In another work, we demonstrated the role of melatonin, together with other molecules as hyaluronic, butyric and retinoic acid in inducing an osteogenic phenotype in dental pulp derived stem cells [18]. It is well known that adipogenic and osteogenic differentiation represent opposite fate, which could be influenced by external stimuli [19].

Aim of the present study was to dissect the role of melatonin with or without vitamin $D$ as a physiological agent able to influence stem cell fate. In particular, we used ADSCs cultured in the presence of melatonin, and vitamin $\mathrm{D}$ in an adipogenic medium in the attempt to define the resulting stem cell differentiation process. Here, we also provide evidence for an epigenetic modulation of melatonin, which is able to induce HADC1, SIRT1 and SIRT2 gene expression. Our findings unfold some main mechanisms underlying stem cell differentiation and could open the way to novel regenerative tool acting as epigenetic modulators, finely balancing stem cell fate toward the adipogenic or osteogenic phenotype.

\section{Materials and Methods}

\subsection{Cell isolation and culturing}

Adipose-derived Stem cells (ADSCs) were obtained from omental adipose tissue of human adult patients, males and females, during surgery processes for different reasons $(n=12$, age $=45 \pm 15$ years, BMI: 22 $\pm 3 \mathrm{~kg} / \mathrm{m} 2$ ). The study was approved by the Ethics Committee Review Boards for Human Studies in Sassari (n_ ETIC 240I/CE 26 July 2016, Ethical committee, ASL Sassari). All patients signed written informed consent.

The fat tissue was washed two times with sterile Dulbecco's phosphate buffered saline (DPBS) (Euroclone, Milano, Italy) to remove blood cells and immediately processed. The sample was mechanically reduced to small fragments by sterile scalpels and enzymatically digest in a solution of $0,1 \%$ Collagenase Type I (Gibco Life Technologies, Grand Island, NY, USA) at $37^{\circ} \mathrm{C}$ for 1 hour, to separate the two principal cell populations of mature adipocytes, that were removed, and the stromal vascular fraction (SVF) that includes adipose-derived stem cells [20]. At the end of incubation, to separate the different cell fractions, samples were filtered in $70 \mu \mathrm{m}$ cell strainer (Euroclone, Milano, Italy) and centrifuged $10 \mathrm{~min}$ at $600 \mathrm{~g}$. The pellet of SVF was resuspended into a basic Dulbecco's modified Eagle's Medium (DMEM) (Life Technologies Grand Island, NY, USA) supplemented with 20\% fetal bovine serum (FBS) (Life Technologies, Grand Island, NY, USA), 200mM L-glutamine 
(Euroclone, Italy), and $200 \mathrm{U} / \mathrm{mL}$ penicillin -0.1 $\mathrm{mg} / \mathrm{mL}$ streptomycin (Euroclone, Milano, Italy) and transferred in incubator at $37^{\circ} \mathrm{C}$ and $5 \% \mathrm{CO} 2$. The culture medium was changed every 3 days.

ADSCs at confluence were counted and then magnetically separated from the stromal vascular fraction and characterized by flow cytometry as previously described [17].

One group of cells were maintained in a growth basic medium (BM) used as undifferentiated control. To define stem cell differentiation processes, ADSCs at passage 5 were grown in an adipose differentiation medium (ADM) (EUROMED Human Adipogenic differentiation Kit) (Euroclone, Milano, Italia) supplemented with $200 \mathrm{U} / \mathrm{mL}$ penicillin- $0.1 \mathrm{mg} / \mathrm{mL}$ streptomycin (Euroclone, Milano, Italy). Another group of cells were exposed to the ADM with the addition of $0,01 \mathrm{M}$ Melatonin (Melatonin+ADM) or the ADM with the addition of melatonin plus $10^{-6} \mathrm{M}$ Vitamin D (Melatonin+VitaminD+ADM) as previously described [17]. As a positive control for osteogenic differentiation were used ADSCs cultured in a specific osteogenic differentiation medium (ODM) containing DMEM (Life Technologies Grand Island, NY, USA), 20\% FBS (Life Technologies, Grand Island, NY, USA), $100 \mathrm{nM}$ dexamethasone, $200 \mu \mathrm{M}$ L-Ascorbic acid 2-phosphate, $10 \mathrm{mM}$ betaglycerol2-phosphate (all from Sigma Aldrich Chemie $\mathrm{GmbH}$, Munich, Germany), $2 \mathrm{mM}$ L-glutamine (Euroclone, Milan, Italy), $200 \mathrm{U} / \mathrm{mL}$ penicillin- $0.1 \mathrm{mg} / \mathrm{mL}$ streptomycin (Euroclone, Milan, Italy), as previously described [18].

\subsection{RNA Extraction and Quantitative Polymerase Chain Reaction}

Total RNA was extracted from undifferentiated cells and ADSCs committed to the adipogenic and osteogenic phenotype in the presence of the previously described conditions, at days 3, 7, 14 and 21, using TRIzol Reagent (Thermo Fisher Scientific) according to the manufacturer's instructions. Total
RNA was measured by spectroscopy with Nanodrop ND-1000 UV-Vis Spectrophotometer (Nanodrop Technologies, Wilmington, DE) to determine purity and concentration. For cDNA synthesis, $1.5 \mu \mathrm{g}$ of RNA from each treatment was reverse transcribed using the Superscript Vilo cDNA synthesis kit (Life Technologies USA), according to the manufacturer's protocol.

Quantitative real-time PCR was performed using a CFX Thermal Cycler (Bio-Rad). Each sample was conducted in triplicate wells with all genes including endogenous control and non-template control on the same plate.

Amplification were run in 96-well reaction plates (Applied Biosystems, Darmstadt, Germany) using Platinum ${ }^{\circledR}$ Quantitative PCR SuperMix-UDG Kit (Thermo Fisher Scientific). The reaction mix contained, in $25 \mu \mathrm{L}$ volumes, $3 \mu \mathrm{L}$ cDNA generated from $1.5 \mu \mathrm{g}$ of the total RNA template, forward primer $(0.5 \mu \mathrm{M})$, reverse primer $(0.5 \mu \mathrm{M}), 2 \times$ SuperMix whit SYBR Green I. Preincubation was performed $50{ }^{\circ} \mathrm{C}$ for $2 \mathrm{~min}, 95 \circ \mathrm{C}$ for $2 \mathrm{~min}$ followed by 40 cycles of $30 \mathrm{secs}$ at $95^{\circ} \mathrm{C}$ for denaturation, 30 secs at $60-64$ for annealing and $1 \mathrm{~min}$ at $60^{\circ} \mathrm{C}$ for extension.

The relative expression of each transcript was determined using target $\mathrm{Ct}$ values and normalized to hGAPDH, considered as a reference gene, while the mRNA levels of ADSCs treated with the different conditioned media were expressed as fold of change $(2-\Delta \Delta \mathrm{Ct})$ of the mRNA levels observed in undifferentiated ADSCs at time 0, define as a control. The qRT-PCR analysis was performed for the following set of genes: histone deacetylases class I (HDAC1) histone deacetylases class III or Sirtuins (SIRT 1 and 2), Stanniocalcin 1 (STC1), Osteocalcin (bone 0gamma-carboxyglutamic acid-containing protein BGLAP), Bone morphogenetic protein (BMP2) and peroxisome proliferator-activated receptor gamma (PPAR- $\gamma)$. All primers used were from Life Technologies and are reported in Table 1.

Table 1. Primers sequences.

\begin{tabular}{l|l|l}
\hline \multicolumn{1}{c|}{ Primers } & \multicolumn{1}{c}{ Forward } & \multicolumn{1}{c}{ Reverse } \\
\hline hGAPDH & GAGTCAACGGATTTGGTCGT & GACAAGCTTCCCGTTCTCAG \\
BMP2 & GAAGAAGAGGAGACTTCAAATG & TATCCCCAGCCTTCTTGGGA \\
BGLAP & GAGCCCCAGTCCCCTACCCG & GACACCCTAGACCGGGCCGT \\
STC1 & TTCGGAGGTGCTCCACTTC & CAGGCTTCGGACAAGTCTGT \\
PPAR- $\gamma$ & AATCCGTCTTCATCCACAGG & GTGAAGACCAGCCTCTTGC \\
HDAC1 & ACTGCTAAAGTATCACCAGAGGG & CACACTTGGCGTGTCCTTG \\
SIRT1 & CATITCCATGGCGCTGAGG & TGCTGGTGGAACAATTCCTGT \\
SIRT2 & TTGCTGAGCTCCTTGGATGG & GGGGAGGGAGCTGTAAGAGA \\
\hline
\end{tabular}


BM

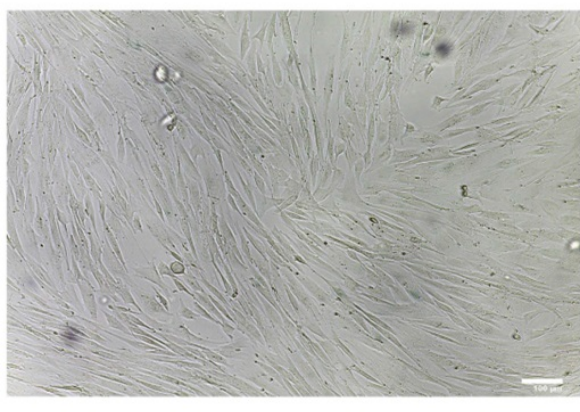

ADM

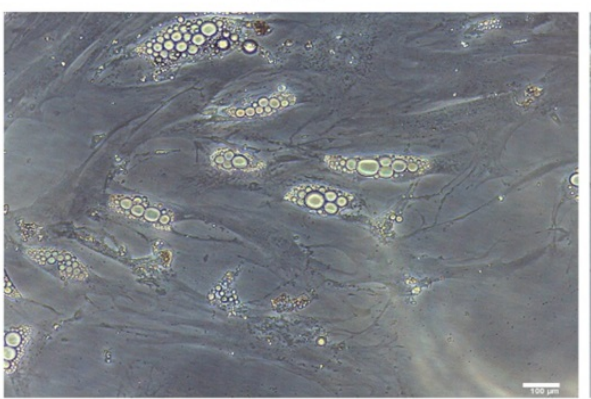

Figure 1. Optical microscope analysis of ADSC morphology during differentiation. Figure shows morphological changes in cell treated with differentiation medium in the presence of melatonin (Melatonin+ADM) or both melatonin and vitamin D (Melatonin+VitaminD+ADM), compared with undifferentiated cells (BM). ADSCs cultured in adipogenic medium alone acquired the appearance of mature adipocytes (ADM). Scale bar=100 $\mu \mathrm{m}$.

\subsection{Alizarin Red Assay}

Cells were cultured for 21 days on tissue culture plate 24 wells (BD-falcon), in the presence of one of differentiation media (ADM or Melatonin+ADM or Melatonin+VitaminD+ADM). Control undifferentiated cells were cultured in the presence of only basic medium. Positive control (CTRL+) was represented by ADSCs cultured in osteogenic medium. Samples were fixed with $10 \%$ formalin for $15 \mathrm{~min}$ at RT, washed three times in distilled water (ddH2O), and then were stained with $2 \%$ alizarin red $\mathrm{S}$ solution (Santa Cruz Biotechnology) for 20min at RT. Cells were thoroughly washed several times in $\mathrm{ddH} 2 \mathrm{O}$ to avoid excess of solution and observed by light microscopy to analyze calcium deposition. The analysis of mineralization was performed using image analysis software (ImageJ, National Institutes of Health).

\subsection{Statistical Analysis}

Data were analyzed using Statistical Package for the Social Sciences version 13 Software (SPSS Inc., Chicago, IL, USA). Krustal-Wallis rank sum and Wilcoxon signed-rank test were applied to evaluate the distributions of each group variance at different times of observation, assuming $p$ value $<0.05$ as statistically significant.

\section{Results}

\subsection{Morphological features of ADSCs cultured in different condition}

After 21 days of differentiation, ADSC morphology was evaluated by optical microscopy (Leica, Nussloch, Germany). We observed significant changes in morphology of ADSCs treated with adipose differentiation medium and melatonin (Figure 1, Melatonin+ADM) or in cells treated in adipose differentiation medium with both melatonin and vitamin D (Figure 1, Melatonin+ VitaminD+ADM), compared with undifferentiated cells (Figure 1, BM). The same figure shows that ADSCs cultured in adipogenic medium alone exhibited a typical morphology of mature adipocytes (Figure 1, ADM).

\subsection{Melatonin and Vitamin $D$ induce the molecular pattern of osteogenesis}

Figure 2 shows the expression of the osteogenic related genes Stanniocalcin (STC1) (Figure 2, A), Osteocalcin (BGLAP) (Figure 2, B) and Bone morphogenetic protein (BMP2) (Figure 2, C). All the analyzed genes are not expressed in ADSCs cultured in the adipogenic medium, while they are significantly induced when melatonin was added to the differentiation medium. Interestingly, the gene expression of all the osteogenic related genes was further upregulated when cells were cultured in the adipogenic medium together with melatonin plus vitamin D (Figure 2). On the other hand, the specific master regulator of adipogenic differentiation, PPAR- $\gamma$, showed an upregulation in ADSCs cultured in a specific adipogenic differentiation medium, while was dramatically decreased when cells were cultured for 14 and 21 days in an adipogenic medium in the presence of melatonin and vitamin D (Figure 2, D).

\subsection{Melatonin and Vitamin D induce the appearance of an osteogenic phenotype}

Consistently with the gene expression analyses of the main markers of osteogenic commitment, we found that melatonin was able to induce cytosolic calcium accumulation, despite the presence of an 
adipogenic-conditioned medium (Figure 3, Melatonin+ADM). As expected, cells grown in an adipogenic differentiation medium did not show any accumulation but they assumed the appearance of mature adipocyte (Figure 3, ADM). The mineralization process and calcium accumulation detected was higher when ADSCs were cultured in the differentiation medium with both melatonin and vitamin D (Figure 3, Melatonin+VitaminD+ADM).

\subsection{Melatonin with or without vitamin D induce HDACl and Sirtuins gene expression}

Figure 4 shows the expression of HDAC1 (Figure 4, A) and SIRT1 (Figure 4, B) and 2 (Figure 4, C), in ADSCs cultured in the previously described conditions and in osteogenic medium, as a positive control. All of the three genes were expressed in cells exposed to the adipogenic medium, but in the presence of melatonin, they were significantly upregulated, reaching a maximum after 21 days in culture (Figure 4). Interestingly, when also vitamin D was added to the differentiation medium (Figure 4, red bar), the mRNA levels of HDAC1, SIRT1 and 2 significantly increased as compared to cells exposed to the adipogenic medium alone, or to the adipogenic medium containing melatonin. HDAC1, SIRT1 and 2 gene expression detected in ADSCs exposed to the classical osteogenic medium, exhibited the same trend previously described for the ADSCs exposed to the three different differentiation media (ADM; Melatonin+ADM; Melatonin+VitaminD+ADM), demonstrating a role of these epigenetic regulating genes in the osteogenic differentiation.

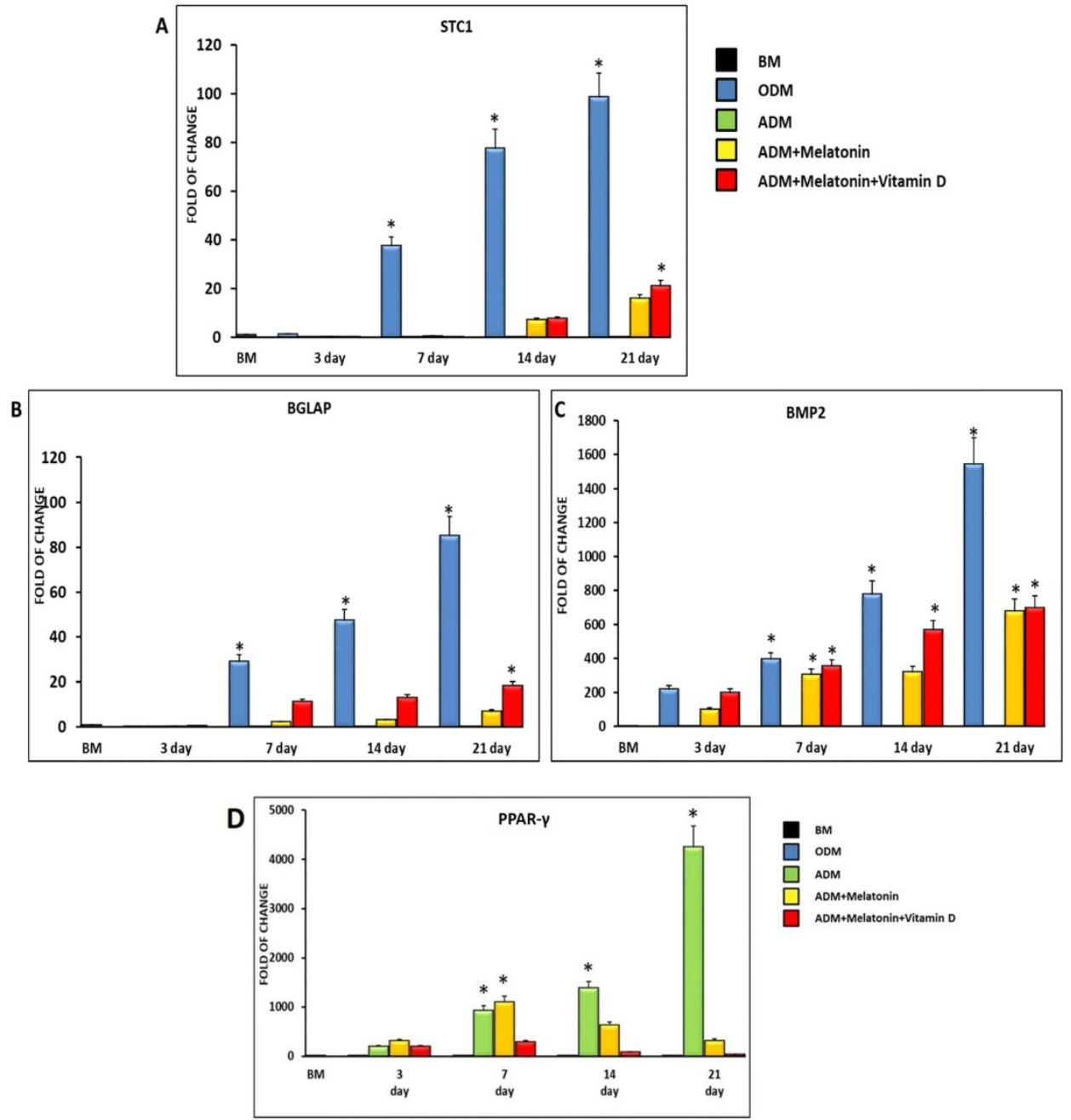

Figure 2. Expression of osteogenic and adipogenic regulating gene. The expression of the osteogenic related genes Stanniocalcin (STCl) (Panel A), Bone morphogenetic protein (BMP2) (Panel B) and Osteocalcin (BGLAP) (Panel C) and of the adipogenic regulating gene PPAR-y (Panel D), was evaluated in ADSCs cultured in adipogenic differentiation medium (green bar), or in adipogenic differentiation medium in the presence of melatonin (yellow bar) or in differentiation medium with melatonin plus vitamin D (red bar). The mRNA levels for each gene were normalized to Glyceraldehyde-3-Phosphate-Dehidrogenase (GAPDH) and expressed as fold of change $(2-\Delta \Delta C t)$ of the mRNA levels observed in undifferentiated ADSCs (black bar) defined as 1 (mean $\pm S D ; n=6$ ). ADSCs cultured in osteogenic conditioned medium represented the positive control (blue bar). Data from differentiation medium together melatonin and vitamin $\mathrm{D}$ show upregulation of osteogenic genes at 14 days and were significantly different after 21 days compared with the differentiation medium alone. Data are expressed as mean \pm SD referred to the control $(* p \leqslant 0.05)$. 
A

BM
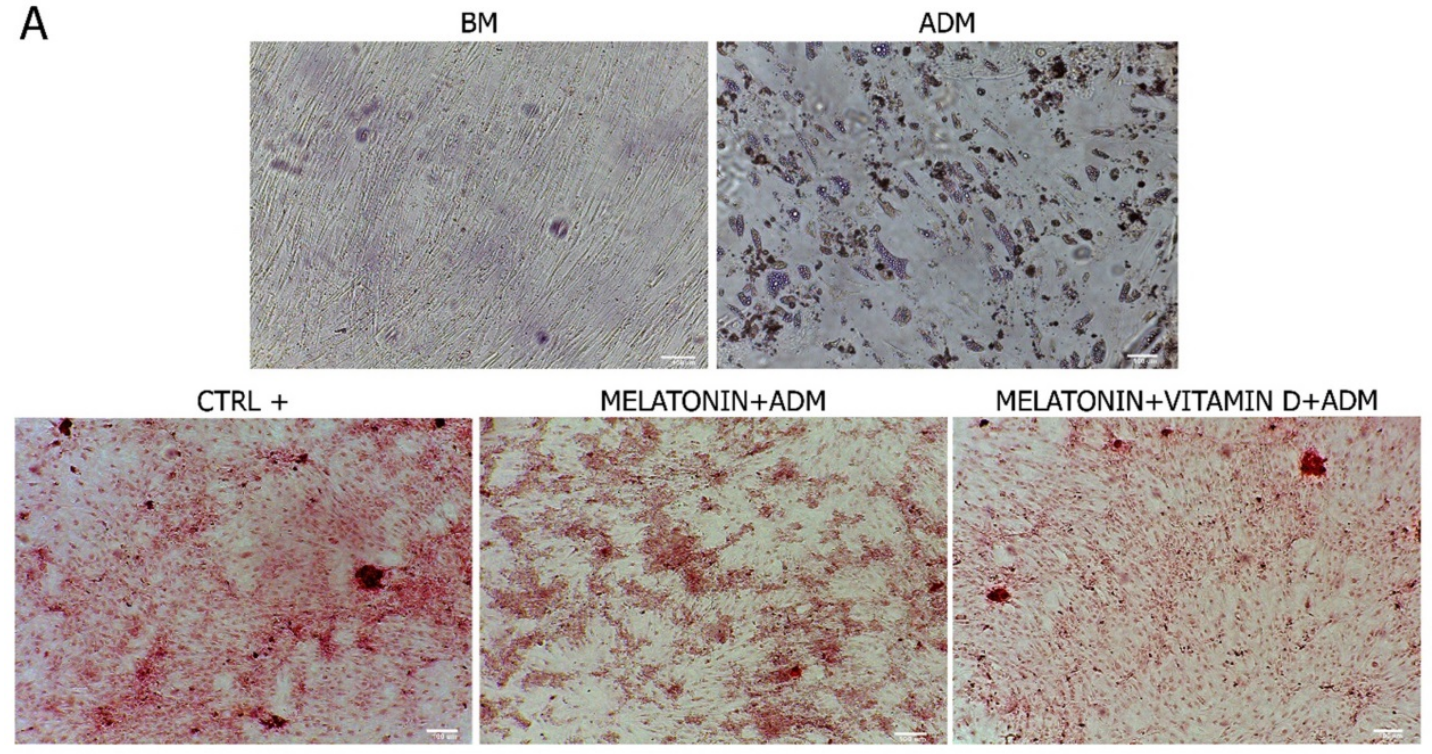

B Alizarin Red Assay at 21 days

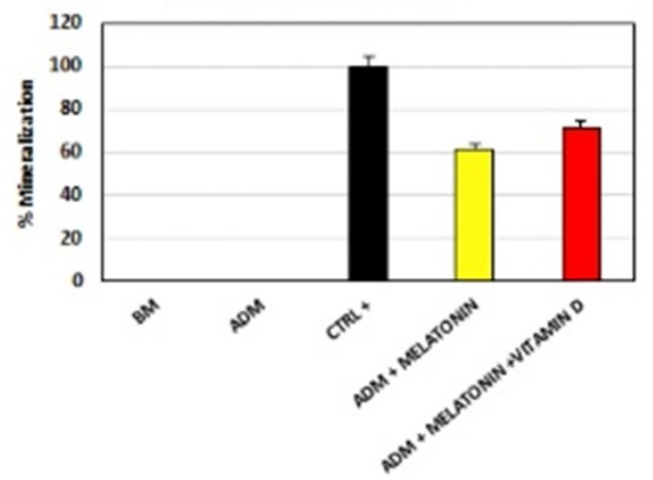

Figure 3. Effect of melatonin and vitamin D on calcium accumulation in ADSCs during differentiation. (A) calcium accumulation, after 21 days of treatment, in cells cultured in basic medium (BM) and ADSCs exposed to differentiation medium (ADM) or ADM together Melatonin (Melatonin+ADM) or both Melatonin and vitamin $D$ (Melatonin+VitaminD+ADM). Positive control (CTRL+) are ADSCs cultured in osteogenic conditioned medium. Scale bar=100 $\mu$ m. The percentage of mineralization (B) was calculated using Imagej, with ADSCs cultured for 21 days in osteogenic medium as positive control (black bar), considered as 1 , and ADSCs cultured in basic medium (white bar) as negative control for calcium accumulation. ADSCs were exposed for 21 days in the presence of ADM only (Blue bar), or ADM with Melatonin (yellow bar) or with both Melatonin and Vitamin D (red bar). Data are expressed as mean \pm SD and are representative of 6 different experiments. An average was made from three technical replicates.

\section{Discussion}

Epigenetic modulators as for example butyric acid have been previously used to orchestrate stem cell fate. In particular, our group previously demonstrated the capability of butyric acid, acting as an inhibitor of HDAC, in a mixture with retinoic and hyaluronic acids to obtain a high yield of cardio-myocyte differentiation in murine embryonic stem cells and in human mesenchymal stem cells, obtained from different sources [21][22][23].

The same mixture of compounds induced the appearance of an osteogenic phenotype in dental-pulp-derived stem cells, when melatonin was added to the differentiation medium [18]. More recently, we demonstrated a major role of Hyaluronic acid in counteracting stem cell senescence obtained after prolonged passages [24], further compounding the multifaceted action of this glycosaminoglycan [25].

Adipogenic differentiation represents a favorite fate for adipose derived stem cells, at the expense of the osteogenic phenotype [26]. We have previously shown that melatonin together with vitamin D was able to counteract adipogenic differentiation, even in the presence of an adipogenic differentiation medium [17]. In the present study, we aimed at defining which phenotype was acquired by cells cultured in these defined conditions. As indicated in figure 2, the molecular pathway controlling the osteogenic fate was induced in our culturing medium based on melatonin, vitamin D and a standard adipogenic medium. Osteogenic differentiation was further inferred by Alizarin red assay, indicating high 
calcium deposition in cells cultured in adipogenic medium in the presence of both melatonin and vitamin D (figure 3). HDACs are a group of enzymes controlling the chromatin state [27], by removing acetyl groups from histones, and thus repressing transcription [28]. Among the two families of HDACs, Class I $(1,2,3,8)$ and Class II $(4,5,6,7,9,10)$, HDAC 1 have been previously described as regulators of stem cell pluripotency in human cardiac mesenchymal stromal cells, with their inhibition inducing an overexpression of Oct4, Sox2, Nanog and Klf4 genes[29].

In the present paper, we show that during the first three days of cell exposure to all the differentiating conditions, HDACs 1 is downregulated (figure 4). After 7 days of culturing, HDACs is upregulated in all differentiating conditions. In particular the medium containing melatonin and vitamin $\mathrm{D}$ exhibited the higher gene expression levels (figure 4) confirming that this gene is upregulated during osteogenenis. Our results describe a role of HDACs 1 during the osteogenic differentiation process, and are coherent with previous observations by other Authors, describing an inhibition of HDACs during the first days of differentiation toward the osteogenic phenotype [30]. The same Authors highlight an activation of the same HDACs 1 to obtain an adipogenic phenotype [31]. In the present study, the culture conditions are actually favoring adipogenesis, inducing HDAC1, while in the presence of melatonin we show that HDACs gene expression is downregulated as compared to the adipogenic medium alone. However, when also vitamin D was added to the culture medium, HDACs was dramatically induced starting from day 7 . Considering our results and what previously shown by other Authors about the HDAC regulation of pluripotency [29], we can hypothesize that the upregulation of HDAC gene expression described by us could explain the establishment of an adipogenic commitment at the beginning, followed by an osteogenic differentiation and a loss of stemness which is described when mesenchymal stem cells acquire a specific phenotype [32].
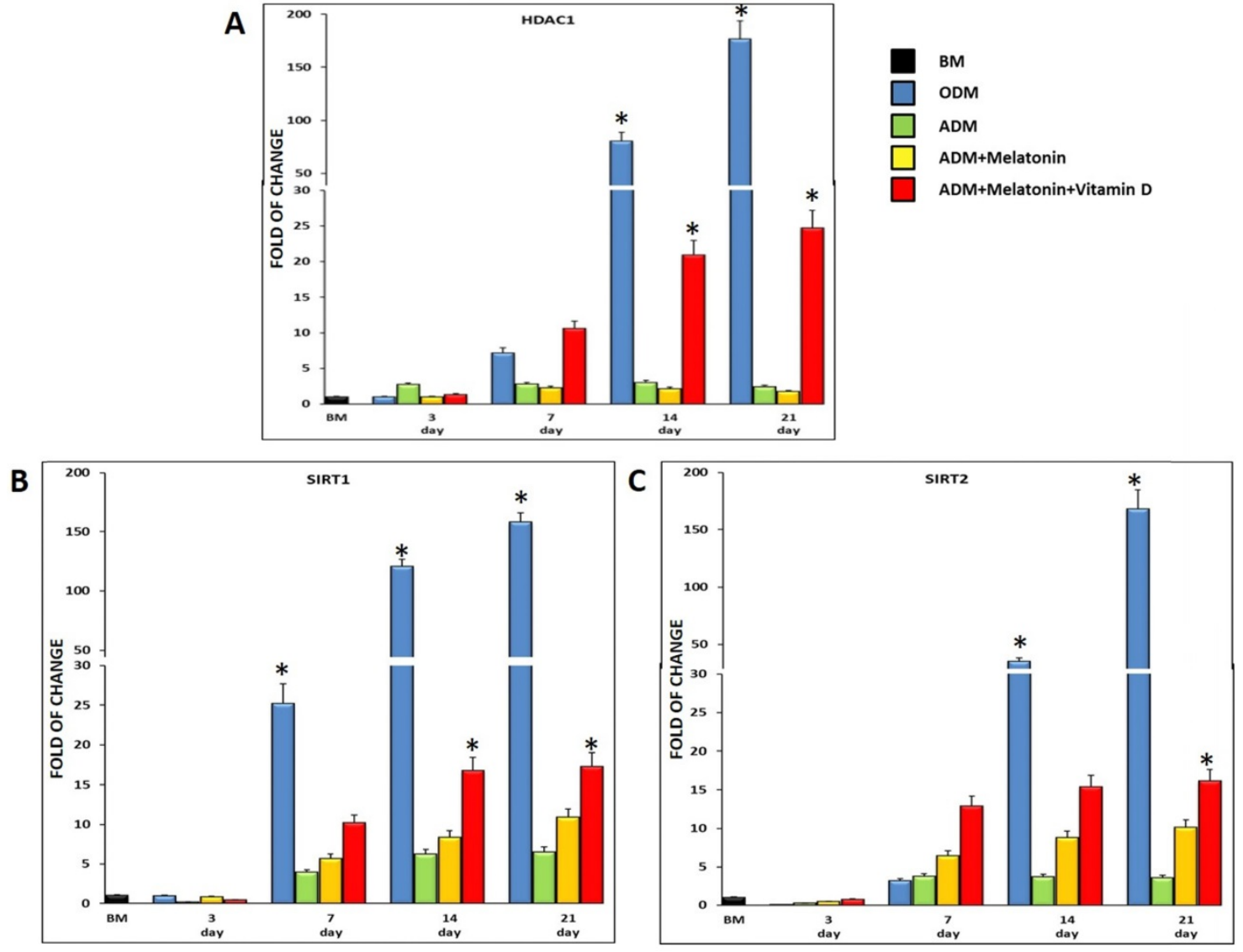

Figure 4. $\mathrm{HDACl}$ and Sirtuins expression in ADSCs cultured in differentiation medium (green bar), or in differentiation medium in the presence of melatonin (yellow bar) or in differentiation medium with melatonin plus vitamin $D$ (red bar). ADSCs (blue bar) cultured in osteogenic medium were used as an osteogenic positive control. The mRNA levels for each gene were normalized to Glyceraldehyde-3-Phosphate-Dehidrogenase (GAPDH) and expressed as fold of change (2- $\Delta \Delta C \mathrm{C}$ ) of the mRNA levels observed in undifferentiated ADSCs (black bar) defined as 1 (mean \pm SD; $n=6$ ). The mRNA levels of HDACl, SIRTI and 2 significantly increased as compared to cells exposed to the adipogenic medium alone, especially when vitamin $D$ was added at differentiation medium together melatonin. Data are expressed as mean $\pm S D$ referred to the control $(* p \leqslant 0.05)$. 

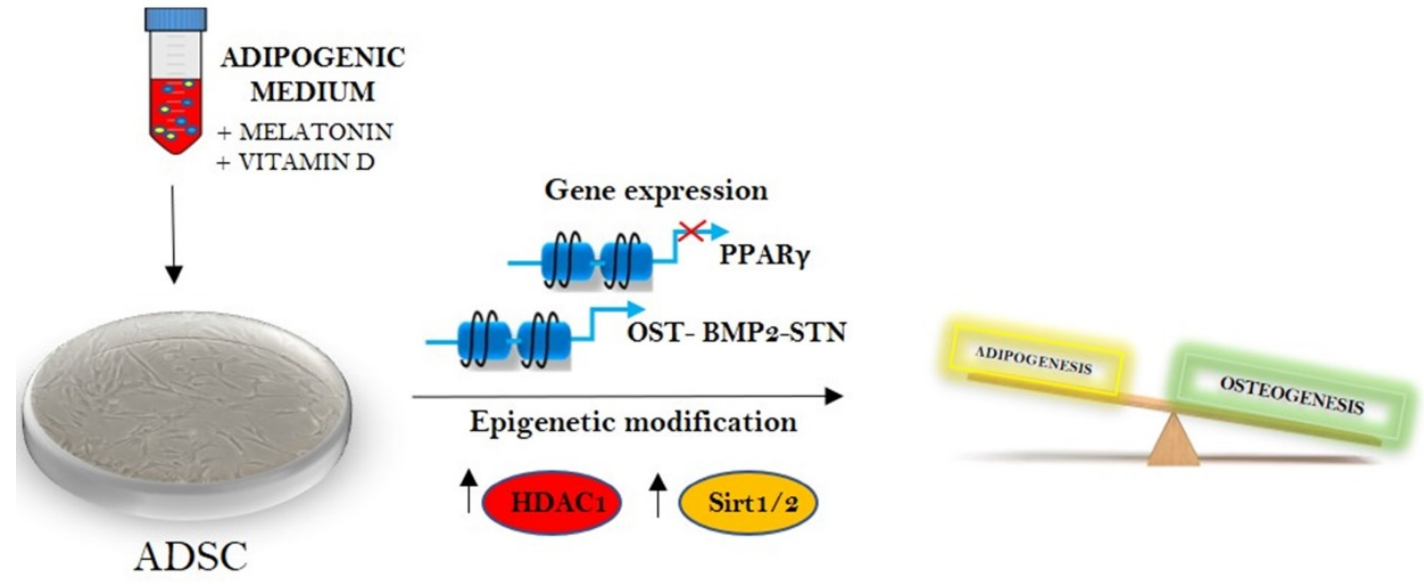

Figure 5. Melatonin and vitamin D in epigenetic regulation of stem cells fate.

The NAD-dependent protein deacetylase SIRT1 has been shown to decrease the appearance of an adipose phenotype in preadipocytes, by inhibiting the PPAR- $\gamma$ [33]. In the present paper, we demonstrate that SIRT1 and 2 are upregulated in the presence of the adipogenic medium, but are overexpressed to a greater extent in the presence of melatonin, and melatonin plus vitamin D (figure 4). Adipocytes and osteocytes share a common stem cell progenitor expressing PPAR- $\gamma$, which is than downregulated when adipose derived stem cells undergo osteogenic differentiation [34]. In this regard we have previously shown that melatonin together with vitamin $\mathrm{D}$ were able to downregulate the expression of the PPAR- $\gamma$ gene (figure 5) even in the presence of an adipogenic conditioned medium [17]. Moreover, we also show that in the presence of an osteogenic medium, ADSCs exhibit the same trend in both HDAC 1 and SIRT1 and 2 gene expression along with the analysed time course (figure 4, blue bars).

Melatonin was first described as a hormone produced by the pineal gland at night, regulating not only circadian rhythm and reproduction, but having a number of pleiotropic effects and whose synthesis decay with aging [35]. Sirtuin 1 and 2 are epigenetic regulators also involved in counteracting a molecular program of aging, which can be induced by different bioactive compounds as for example resveratrol or curcumin [36][37].

Nutritional epigenetics represent a novel branch of science, addressing how bioactive molecules or mixture of bioactive compounds can influence gene expression [38], stem cell behavior, pluripotency and differentiation, but also cancer cell proliferation [39]. In this regard, we have recently shown that a butyric derivative of Honokiol, the main bioactive component of Magnolia Obovata, was found effective in counteracting tumoral hepatocytes cells proliferation while fibroblast cells, used as control for the toxicological effect of the drugs, were unaffected [39]. Even in this pro-apoptotic and pro-autophagic effect of the synthetic compound the activation of SIRT3 seems to be crucial [40].

Also Vitamin D has recently emerged as an important metabolic regulator, able to affect not only calcium absorption, but also many cellular processes, by modulating enzymes responsible for chromatin modification [41]. This molecule is able to affect chromatin remodeling by modulating HDACs and DNA methylation [42].

The results exposed in the present paper for the first time describe a direct link between melatonin and HDAC and melatonin and Sirtuins in adipose derived stem cells (figure 5). The effect elicited by melatonin on these HDACs could represent a perfect circuit embedding Sirtuin activated melatonin and the adipogenic differentiation arrest induced by the indole, defining future application of this drug for the weight control.

\section{Acknowledgements}

The authors are grateful for the financial support from Eldor Lab SRL, Milan, Italy and Fondazione di Sardegna, Project Prot. U858.2014/AI.741.MGB Prat.2014.0178 and Project Prot. U933.2018/AI.907.RP Prat.2018.1912.

\section{Author Contributions}

Sara Santaniello and Sara Cruciani contributed equally to this work. Sara Santaniello, Sara Cruciani, Carlo Ventura and Margherita Maioli conceived the idea of the article, designed the experimental plan and wrote the paper. Valentina Basoli, Francesca Balzano, Emanuela Bellu wrote the paper and reviewed scientific literature. Sara Santaniello and Sara Cruciani performed the experiments and wrote the paper. Giorgio Carlo Ginesu, Maria Laura Cossu, Alessandro P. Delitala and Federica Facchin participated in 
sample collection and in figure preparation. All the authors gave the final approval of the version to be submitted.

\section{Conflicts of Interest}

The authors declare no conflict of interest.

\section{References}

1. Guilak F, Cohen DM, Estes BT, et al. Control of Stem Cell Fate by Physical Interactions with the Extracellular Matrix. Cell Stem Cell. 2009; 5(1):17-26.

2. Bianchi $\mathrm{F}$, et al. A new nonenzymatic method and device to obtain a fat tissue derivative highly enriched in pericyte-like elements by mild mechanical forces from human lipoaspirates. Cell Transplant. 2013; 22(11):2063-77.

3. Ma Y, et al. Melatonin Ameliorates Injury and Specific Responses of Ischemic Striatal Neurons in Rats. J. Histochem. Cytochem. 2013; 61(8):591-605.

4. Jenwitheesuk A, Nopparat C, et al. Melatonin regulates aging and neurodegeneration through energy metabolism, epigenetics, autophagy and circadian rhythm pathways. International Journal of Molecular Sciences. 2014; 15(9):16848-84

5. Loureiro et al. Melatonin antiproliferative effects require active mitochondrial function in embryonal carcinoma cells. Oncotarget. 2015; 6(19):17081-96.

6. Legros C, et al. Melatonin MT1 and MT2 receptors display different molecular pharmacologies only in the G-protein coupled state. Br. J. Pharmacol. 2014; 171(1): 186-201.

7. Sharma R, Ottenhof $\mathrm{T}$, et al. Epigenetic targets for melatonin: Induction of histone H3 hyperacetylation and gene expression in C17.2 neural stem cells. J. Pineal Res. 2008; 45(3):277-84.

8. Kupis W, Pałyga J, et al. The role of sirtuins in cellular homeostasis. Journal of Physiology and Biochemistry. 2016; 72(3):371-80

9. Fiorino E, et al. The sirtuin class of histone deacetylases: Regulation and roles in lipid metabolism. IUBMB Life. 2014; 66(2):89-99.

10. Choudhary C, Weinert BT, et al. The growing landscape of lysine acetylation links metabolism and cell signalling. Nature Reviews Molecular Cell Biology. 2014; 15(8):536-50.

11. Poulose N, Raju R. Sirtuin regulation in aging and injury. Biochimica et Biophysica Acta - Molecular Basis of Disease. 2015; 1852(11):2442-55.

12. Grabowska W, Sikora E, Bielak-Zmijewska A. Sirtuins, a promising target in slowing down the ageing process. Biogerontology. 2017; 18(4):447-476.

13. Tarantino G, et al. Circulating levels of sirtuin 4, a potential marker of oxidative metabolism, related to coronary artery disease in obese patients suffering from nafld, with normal or slightly increased liver enzymes. Oxid. Med. Cell. Longev. 2014; 2014:920676.

14. Liu Z, Gan L, Zhang T, et al. Melatonin alleviates adipose inflammation through elevating a-ketoglutarate and diverting adipose-derived exosomes to macrophages in mice. J. Pineal Res. 2018; 64(1)

15. Wolk R, Shamsuzzaman ASM, Somers VK. Obesity, Sleep Apnea, and Hypertension. Hypertension. 2003; 42(6):1067-74.

16. Cipolla-Neto J, Amaral FG, et al. Melatonin, energy metabolism, and obesity: A review. Journal of Pineal Research. 2014; 56(4):371-81.

17. Basoli $\mathrm{V}$, et al. Melatonin and vitamin $\mathrm{D}$ interfere with the adipogenic fate of adipose-derived stem cells. Int. J. Mol. Sci. 2017; 18(5).

18. Maioli M, et al. Osteogenesis from Dental Pulp Derived Stem Cells: A Novel Conditioned Medium Including Melatonin within a Mixture of Hyaluronic, Butyric, and Retinoic Acids. Stem Cells Int. 2016; 2016:2056416.

19. Huang $\mathrm{S}$, et al. Upregulation of miR-22 promotes osteogenic differentiation and inhibits adipogenic differentiation of human adipose tissue-derived mesenchymal stem cells by repressing HDAC6 protein expression. Stem Cells Dev. 2012; 21(13):2531-40.

20. Bora P, Majumdar AS. Adipose tissue-derived stromal vascular fraction in regenerative medicine: a brief review on biology and translation. Stem Cell Res. 2017; 8(1):145

21. Ventura $C$, et al. Butyric and retinoic mixed ester of hyaluronan: A novel differentiating glycoconjugate affording a high throughput of cardiogenesis in embryonic stem cells. J. Biol. Chem. 2004; 279(22):23574-9.

22. Maioli $\mathrm{M}$, et al. Hyaluronan esters drive smad gene expression and signaling enhancing cardiogenesis in mouse embryonic and human mesenchymal stem cells. PLoS One. 2010; 5(11):e15151.

23. Maioli M, et al. Amniotic fluid stem cells morph into a cardiovascular lineage: Analysis of a chemically induced cardiac and vascular commitment. Drug Des. Devel. Ther. 2013; 7:1063-73.

24. Maioli M, et al. REAC technology and hyaluron synthase 2, an interesting network to slow down stem cell senescence. Sci. Rep. 2016; 6:28682.

25. Kothapalli D, Flowers J, et al. Differential activation of ERK and Rac mediates the proliferative and anti-proliferative effects of hyaluronan and CD44. J. Biol. Chem. 2008; 283(46):31823-9.

26. Kocan B, Maziarz A, et al. Trophic Activity and Phenotype of Adipose Tissue-Derived Mesenchymal Stem Cells as a Background of Their Regenerative Potential. Stem Cells International. 2017; 2017:1653254.
27. Haggarty SJ, Tsai LH. Probing the role of HDACs and mechanisms of chromatin-mediated neuroplasticity. Neurobiol. Learn. Mem. 2011; 96(1):41-52.

28. Hull EE, Montgomery MR, Leyva KJ. HDAC Inhibitors as Epigenetic Regulators of the Immune System: Impacts on Cancer Therapy and Inflammatory Diseases. BioMed Research International. 2016; 2016:8797206.

29. Moore JB, et al. Histone deacetylase 1 depletion activates human cardiac mesenchymal stromal cell proangiogenic paracrine signaling through a mechanism requiring enhanced basic fibroblast growth factor synthesis and secretion. J. Am. Heart Assoc. 2017; 6(7).

30. Huynh NCN, Everts V, Ampornaramveth RS. Histone deacetylases and their roles in mineralized tissue regeneration. Bone Reports. 2017; 7:33-40.

31. Lee $\mathrm{S}$, et al. Histone deacetylase inhibitors decrease proliferation potential and multilineage differentiation capability of human mesenchymal stem cells. Cell Prolif. 2009; 42(6):711-20.

32. Maioli $\mathrm{M}$, et al. Radioelectric asymmetric conveyed fields and human adipose-derived stem cells obtained with a nonenzymatic method and device: A novel approach to multipotency. Cell Transplant. 2014; 23(12):1489-500.

33. Picard F, et al. Sirt1 promotes fat mobilization in white adipocytes by repressing PPAR-ү. Nature. 2004; 429(6993):771-6.

34. Bäckesjö CM, Li Y, et al. Activation of Sirt1 decreases adipocyte formation during osteoblast differentiation of mesenchymal stem cells. Cells Tissues Organs. 2009; 189(1-4):93-7

35. Kleszczynski K, Fischer TW. Melatonin and human skin aging. Dermato-Endocrinology. 2012; 4(3): 245-252.

36. Buhrmann C, Shayan P, Popper B, et al. Sirt1 is required for resveratrol-mediated chemopreventive effects in colorectal cancer cells. Nutrients. 2016; 8(3): 145.

37. Shakibaei $M$, et al. Resveratrol mediated modulation of sirt-1/Runx2 promotes osteogenic differentiation of mesenchymal stem cells: Potential role of Runx2 deacetylation. PLoS One. 2012; 7(4)

38. Waterland RA. Nutritional Epigenetics. Present Knowledge in Nutrition: Tenth Edition. 2012.

39. Maioli M, et al. Synthesis of magnolol and honokiol derivatives and their effect against hepatocarcinoma cells. PLoS One. 2018; 13(2):e0192178.

40. Luo LX, et al. Honokiol induces apoptosis, G1 arrest, and autophagy in KRAS mutant lung cancer cells. Front. Pharmacol. 2017; 8:199.

41. Christakos S, Dhawan P, et al. Vitamin D: Metabolism, Molecular Mechanism of Action, and Pleiotropic Effects. Physiol. Rev. 2016; 96(1):365-408.

42. Seuter S, Heikkinen S, Carlberg C. Chromatin acetylation at transcription start sites and vitamin $\mathrm{D}$ receptor binding regions relates to effects of 1a,25-dihydroxyvitamin D3 and histone deacetylase inhibitors on gene expression. Nucleic Acids Res. 2013; 96(1):365-408. 